\title{
ASSOCIATION BETWEEN MENDELIAN FACTORS WITH MIXED SELFING AND RANDOM MATING
}

\author{
J. H. BENNETT \\ Department of Genetics, Cambridge University \\ and \\ F. E. BINET \\ Department of Statistics, Melbourne University
}

Received 20.vii.55

IN most plant species in which reproduction is effected both by crossand self-fertilisation, the relative contributions from these two kinds of matings depend to some extent on climatic and other environmental conditions and so vary from one generation to the next. In the present article, however, we shall confine our attention to the simplified but fundamental situation in which these contributions bear a constant ratio to one another throughout all generations.

Consider a large population of plants reproducing in non-overlapping generations in such a way that there is a constant probability $s$ that any plant will be self-fertilised and a probability $I-s$ that it will cross with some plant chosen at random from the population. We shall suppose that all crosses are equally fertile and all genotypes equally viable. With this system of mating, gene frequency clearly remains constant. Suppose $A$ and $a$ are the genes present at some locus and $p(\mathrm{~A}), p(a),[p(\mathrm{~A})+p(a)=\mathrm{I}]$ their frequencies in the population. If $p_{n}(\mathrm{~A} a)$ denotes the frequency of occurrence of the genotype $\mathrm{A} a$ in the $n$th generation, we have

and for the genotype AA,

$$
p_{n+1}(\mathrm{~A} a)=\frac{1}{2} s p_{n}(\mathrm{~A} a)+2(\mathrm{I}-s) p(\mathrm{~A}) p(a)
$$

Hence, if we put

$$
p_{n+1}(\mathrm{AA})=s\left[p_{n}(\mathrm{AA})+\frac{1}{4} p_{n}(\mathrm{~A} a)\right]+(\mathrm{I}-s) p(\mathrm{~A})^{2} .
$$

$$
\mathrm{K}_{n}=p_{n}(\mathrm{~A} a)-\frac{4(\mathrm{I}-s)}{2-s} p(\mathrm{~A}) p(a)
$$

then

At equilibrium, $\mathrm{K}=\mathrm{o}$,

$$
\mathrm{K}_{n}=\left(\frac{1}{2} s\right)^{n} \mathrm{~K}_{o} .
$$

$$
\begin{aligned}
\text { i.e. } p_{\infty}(\mathrm{A} a)=\lim _{n \rightarrow \infty} p_{n}(\mathrm{~A} a) & =\frac{4(\mathrm{I}-s)}{2-s} p(\mathrm{~A}) p(a) \\
\text { and } p_{\infty}(\mathrm{AA})=\lim _{n \rightarrow \infty} p_{n}(\mathrm{AA}) & =p(\mathrm{~A})\left[\mathrm{I}-\frac{2(\mathrm{I}-s)}{2-s} p(a)\right] \\
& =p(\mathrm{~A})^{2}+\frac{s}{2-s} p(\mathrm{~A}) p(a) .
\end{aligned}
$$

These equilibrium genotypic frequencies are those which would exist in a composite population comprising a random mating component supplying the proportion $\frac{2(\mathrm{I}-s)}{2-s}$ of the whole population and a 
selfing component making up the remainder. Genotypic equilibrium is not attained in one generation as with random mating but is approached at a rate measured by $-\log _{e}\left(\frac{1}{2} s\right)$.

If $B$ and $b$ are two genes present at a second locus and $y$ is the frequency of recombination between the two loci, the frequency of occurrence of a given chromosome or gene-combination such as $\mathrm{AB}$ in the genotypes of the $(n+1)$-th generation is

It can also be shown that

$$
p_{n+1}(\mathrm{AB})=p_{n}(\mathrm{AB})+\frac{1}{2} y\left[p_{n}(\mathrm{~A} b / a \mathrm{~B})-p_{n}(\mathrm{AB} / a b)\right] \text {. }
$$

$$
\begin{aligned}
p_{n+1}(\mathrm{~A} b / a \mathrm{~B})-p_{n+1}(\mathrm{AB} / a b)=\frac{1}{2}(s-2 y)[ & {\left[p_{n}(\mathrm{~A} b / a \mathrm{~B})-p_{n}(\mathrm{AB} / a b)\right] } \\
& +2(\mathrm{I}-s)\left[p(\mathrm{~A}) p(\mathrm{~B})-p_{n}(\mathrm{AB})\right] .
\end{aligned}
$$

Hence, if we put

$$
u_{n}=p(\mathrm{~A}) p(\mathrm{~B})-p_{n}(\mathrm{AB})
$$

and

we have

$$
v_{n}=p_{n}(\mathrm{~A} b / a \mathrm{~B})-p_{n}(\mathrm{AB} / a b),
$$

$$
\left(\frac{u_{n}}{v_{n}}\right)=\mathrm{Z}^{n} \cdot\left(\frac{u_{o}}{v_{o}}\right)
$$

where $\mathrm{Z}$ stands for the matrix

$$
\left(\begin{array}{cc}
\frac{\mathrm{I}}{2(\mathrm{I}-s)} & -\frac{1}{2} y \\
\frac{1}{2}(s-2 y)
\end{array}\right)
$$

The matrix $\mathrm{Z}$ has two positive and unequal latent roots $\lambda$, $\mu$ which satisfy the equation,

The latent roots are therefore

$$
2 z^{2}-(2+s-2 y) z+s(1-2 y)=0 .
$$

$$
\frac{\lambda}{\mu}=\frac{1}{4}\left\{2+s-2 \cdot y \pm \sqrt{ }\left[(2-s-2 y)^{2}+8 s y\right]\right\} .
$$

Clearly, if neither $\mathrm{I}-s$ nor $y$ is zero, $\lambda(>\mu)$ is less than unity and

$$
\begin{aligned}
& \lim _{n \rightarrow \infty} p_{n}(\mathrm{AB})=p(\mathrm{~A}) p(\mathrm{~B}) \\
& \lim _{n \rightarrow \infty}\left[p_{n}(\mathrm{~A} b / a \mathrm{~B})-p_{n}(\mathrm{AB} / a b)\right]=0 .
\end{aligned}
$$

A measure of the rate of approach to equilibrium is given by $-\log _{e} \lambda$ (see table I).

Corresponding with the two latent roots $\lambda$ and $\mu$, there exist two principal linear components of frequency,

$$
\begin{aligned}
\mathrm{L}_{n} & =2(\mathrm{I}-s) u_{n}-(\mathrm{I}-\lambda) v_{n}=\lambda^{n} \cdot \mathrm{L}_{o} \\
\mathrm{M}_{n} & =2(\mathrm{I}-s) u_{n}-(\mathrm{I}-\mu) v_{n}=\mu^{n} \cdot \mathrm{M}_{o}
\end{aligned}
$$

from which the values of $u_{n}$ and $v_{n}$ can readily be determined for any positive integer $n$.

When $s=\mathrm{I}$, i.e. with complete selfing, the latent roots are $\mathrm{I}$ and $\frac{1}{2}-y$, the corresponding principal components of frequency being and

$$
\begin{aligned}
(\mathrm{I}+2 y) u_{n}-y v_{n} & =\text { constant } \\
v_{n} & =\left(\frac{1}{2}-y\right)^{n} \cdot v_{o} .
\end{aligned}
$$

Hence, in this case,

$$
p_{\infty}(\mathrm{AB})=p_{\infty}(\mathrm{AB} / \mathrm{AB})=p_{o}(\mathrm{AB})+\frac{y}{\mathrm{I}+2 y}\left[p_{o}(\mathrm{~A} b / a \mathrm{~B})-p_{o}(\mathrm{AB} / a b)\right]
$$


giving the limiting genotypic frequencies when complete homozygosity has been attained. In general, when $s=\mathrm{I}$,

$$
p_{\infty}(\mathrm{AB}) \neq p(\mathrm{~A}) p(\mathrm{~B}) \text {. }
$$

With complete random mating, i.e. $s=0$, the latent roots are $\mathrm{I}-y$ and $\mathrm{o}$, the corresponding principal components of frequency being

Hence

$$
\begin{aligned}
& u_{n}-\frac{1}{2} y v_{n}=(\mathrm{I}-y)^{n} \cdot\left[u_{0}-\frac{1}{2} y \cdot v_{0}\right] \\
& u_{n}-\frac{1}{2} v_{n}=0 \quad(n>0) .
\end{aligned}
$$

$$
\begin{aligned}
p(\mathrm{~A}) p(\mathrm{~B})-p_{n}(\mathrm{AB})=\frac{1}{2}\left[p_{n}(\mathrm{~A} b / a \mathrm{~B})-p_{n}(\mathrm{AB} / a b)\right] \\
=(\mathrm{I}-y)^{n-1}\left[p(\mathrm{~A}) p(\mathrm{~B})-p_{0}(\mathrm{AB})-\frac{1}{2} p_{o}(\mathrm{~A} b / a \mathrm{~B})+\frac{1}{2} p_{o}(\mathrm{AB} / a b .)\right]
\end{aligned}
$$

\begin{tabular}{|c|c|c|c|c|c|c|c|c|c|c|c|c|c|}
\hline & \multicolumn{12}{|c|}{ Recombination frequency $y$} \\
\hline & & 0.05 & $0 \cdot 10$ & 0.15 & 0.20 & $0 \cdot 25$ & 0.30 & 0.35 & 0.40 & 0.45 & 0.50 & 0.55 & 0.60 \\
\hline 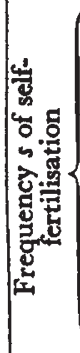 & $\begin{array}{l}0.00 \\
0.05 \\
0.10 \\
0.20 \\
0.30 \\
0.40 \\
0.50 \\
0.60 \\
0.70 \\
0.80 \\
0.90 \\
0.95\end{array}$ & $\begin{array}{l}\cdot 0513 \\
\cdot 0499 \\
\cdot 0484 \\
\cdot 0452 \\
\cdot 0416 \\
\cdot 0376 \\
\cdot 033 \mathrm{I} \\
\cdot .028 \mathrm{I} \\
\cdot 0224 \\
.0159 \\
.0084 \\
.0044\end{array}$ & $\begin{array}{l}\cdot .054 \\
\cdot .022 \\
\cdot 0989 \\
.0918 \\
.0839 \\
.0753 \\
.0657 \\
.0551 \\
.0433 \\
.0303 \\
.0159 \\
.008 \mathrm{r}\end{array}$ & $\begin{array}{l}\cdot 1625 \\
\cdot 1572 \\
\cdot 1517 \\
\cdot 1398 \\
\cdot 1269 \\
\cdot 1129 \\
\cdot 0975 \\
\cdot 0809 \\
\cdot 0628 \\
\cdot 0433 \\
\cdot 0224 \\
\cdot 0114\end{array}$ & $\begin{array}{l}\cdot 2231 \\
\cdot 2152 \\
\cdot 2069 \\
\cdot 1894 \\
\cdot-1705 \\
\cdot 1502 \\
\cdot 1285 \\
\cdot 1054 \\
\cdot 0809 \\
\cdot 0551 \\
.0281 \\
\cdot 0142\end{array}$ & 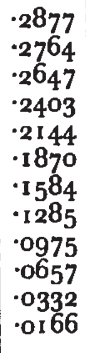 & $\begin{array}{l}\cdot 3567 \\
-3412 \\
\cdot 3253 \\
-2925 \\
\cdot 2584 \\
\cdot 2231 \\
\cdot-1870 \\
\cdot-1502 \\
-1129 \\
\cdot 0753 \\
\cdot 0377 \\
\cdot 0188\end{array}$ & $\begin{array}{l}\cdot 4308 \\
\cdot 4099 \\
\cdot 3888 \\
\cdot 3459 \\
\cdot 3023 \\
\cdot 2584 \\
\cdot 2144 \\
\cdot-1705 \\
\cdot 1270 \\
\cdot 0839 \\
\cdot 0416 \\
.0207\end{array}$ & $\begin{array}{l}\cdot 5108 \\
\cdot 4831 \\
\cdot 4553 \\
\cdot 4002 \\
\cdot 3459 \\
\cdot 2925 \\
\cdot-2403 \\
\cdot 1894 \\
\cdot 1398 \\
\cdot 0918 \\
\cdot 0453 \\
\cdot 0224\end{array}$ & $\begin{array}{l}\cdot 5978 \\
\cdot 56 \mathrm{II} \\
\cdot 5250 \\
\cdot 4553 \\
\cdot 3888 \\
\cdot 3253 \\
\cdot .647 \\
\cdot 2069 \\
\cdot .1517 \\
\cdot 0989 \\
\cdot 0484 \\
\cdot 0239\end{array}$ & $\begin{array}{l}\cdot 6932 \\
\cdot 6444 \\
\cdot 5978 \\
\cdot 5108 \\
\cdot 4308 \\
\cdot 3567 \\
\cdot 2877 \\
\cdot 2231 \\
\cdot .625 \\
\cdot 1054 \\
.0512 \\
.0253\end{array}$ & $\begin{array}{l}\cdot 7985 \\
\cdot 7337 \\
\cdot 6737 \\
\cdot 5664 \\
\cdot 4715 \\
\cdot 3865 \\
\cdot 3092 \\
-2382 \\
-1725 \\
-1113 \\
\cdot 0538 \\
\cdot 0266\end{array}$ & 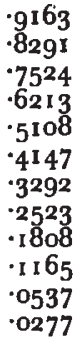 \\
\hline
\end{tabular}

\section{TABLE I}

Rate of approach to equilibrium at two linked loci with recombination frequency $\mathrm{y}$ in a population with $100 \mathrm{~s}$ per cent. self-fertilisation and $100(x-\mathrm{s})$ per cent. random mating

With mixed selfing and random mating $(s \neq 1)$, the genotypic frequencies at equilibrium are given by

$$
\begin{gathered}
p_{\infty}(\mathrm{AB} / a b)=p_{\infty}(\mathrm{A} b / a \mathrm{~B})= \\
4(\mathrm{I}-s) p(\mathrm{~A}) p(a) p(\mathrm{~B}) p(b) /[2-s+2 s y(\mathrm{I}-y)] \\
p_{\infty}(\mathrm{AB} / \mathrm{A} b)=-p_{\infty}(\mathrm{AB} / a b)+4(\mathrm{I}-s) p(\mathrm{~A}) p(a) p(\mathrm{~B}) p(b) /(2-s) \\
p_{\infty}(\mathrm{AB} / \mathrm{AB})= \\
\frac{1}{2} p_{\infty}(\mathrm{AB} / a b)+p(\mathrm{~A}) p(\mathrm{~B})-2(\mathrm{I}-s) p(\mathrm{~A}) p(\mathrm{~B})[p(a)+p(b)] /(2-s) .
\end{gathered}
$$

The equilibrium frequencies for the other genotypes are readily obtainable from these expressions on making appropriate gene substitutions. We see that when equilibrium is-attained, linked factors are associated to an extent that depends not only on the proportion of selfing but also on the magnitude of the recombination fraction. In such a case, it might be possible to determine the recombination 
fraction between two loci from an analysis of the population frequencies. When $y=\frac{1}{2}$, as for two loci on different chromosomes,

$$
\begin{aligned}
& p_{\infty}(\mathrm{A} a \mathrm{~B} b)-p_{\infty}(\mathrm{A} a) p_{\infty}(\mathrm{B} b)=\frac{\mathrm{r} 6 s(\mathrm{I}-s)}{(4-s)(2-s)^{2}} p(\mathrm{~A}) p(a) p(\mathrm{~B}) p(b) \\
& p_{\infty}(\mathrm{AAB} b)-p_{\infty}(\mathrm{AA}) p_{\infty}(\mathrm{B} b)=\frac{-8 s(\mathrm{I}-s)}{(4-s)(2-s)^{2}} p(\mathrm{~A}) p(a) p(\mathrm{~B}) p(b) \\
& p_{\infty}(\mathrm{AABB})-p_{\infty}(\mathrm{AA}) p_{\infty}(\mathrm{BB})=\frac{4 s(\mathrm{I}-s)}{(4-s)(2-s)^{2}} p(\mathrm{~A}) p(a) p(\mathrm{~B}) p(b) .
\end{aligned}
$$

Two factors which segregate independently are thus not associated at random when equilibrium is reached. There is, in fact, a positive association between the homozygous (or heterozygous) states at loci on different chromosomes. This association may be represented as

TABLE 2

Association between the homozygous and heterozygous conditions at two independent loci in a population experiencing mixed selfing and random mating

\begin{tabular}{|c|c|c|c|}
\hline & $\mathrm{AA}$ & $\mathrm{A} a$ & $a a$ \\
\hline $\mathrm{BB}$ & $\mathrm{I}$ & -2 & $\mathrm{I}$ \\
$\mathrm{B} b$ & -2 & 4 & -2 \\
$b b$ & $\mathrm{I}$ & -2 & $\mathrm{I}$ \\
\hline
\end{tabular}

in table 2 which gives the excess of the equilibrium frequencies for genotypes at two independently segregating loci over the product of the equilibrium frequencies at the separate loci as a multiple of

$$
\frac{4 s(\mathrm{I}-s)}{(4-s)(2-s)^{2}} p(\mathrm{~A}) p(a) p(\mathrm{~B}) p(b) \text {. }
$$

For given gene frequencies, this excess has its greatest value

$$
\text { o. I } 506 p(\mathrm{~A}) p(a) p(\mathrm{~B}) p(b)
$$

when $s=0.6946$. (See fig. I.)

There are results analogous to these for randomly mating populations of polysomic organisms where, for our present purposes, double reduction may be considered as equivalent to a small amount of selfing. It has been shown (Bennett, 1954) that at equilibrium in randomly mating populations of tetrasomic or hexasomic organisms the probability of occurrence of any chromosome or gene-combination, such as $\mathrm{AB}$, equals the product of the appropriate gene frequencies, i.e. $p_{\infty}(\mathrm{AB})=p(\mathrm{~A}) p(\mathrm{~B})$. If there is no double reduction, linked loci will therefore be associated at random in the gametes or zygotes at equilibrium. However, when double reduction can occur, linked loci may not be associated at random in the gametes and zygotes at equilibrium though loci on different chromosomes certainly will be. 


\section{SUMMARY}

In a population that experiences mixed selfing and random mating, genes are associated at random on the chromosomes at equilibrium but there is a positive association between the genotypic state (homozygous or heterozygous) at different loci even when the two loci are on different chromosome, i.e. there is a tendency for heterozygosis to become concentrated in some members of the population.

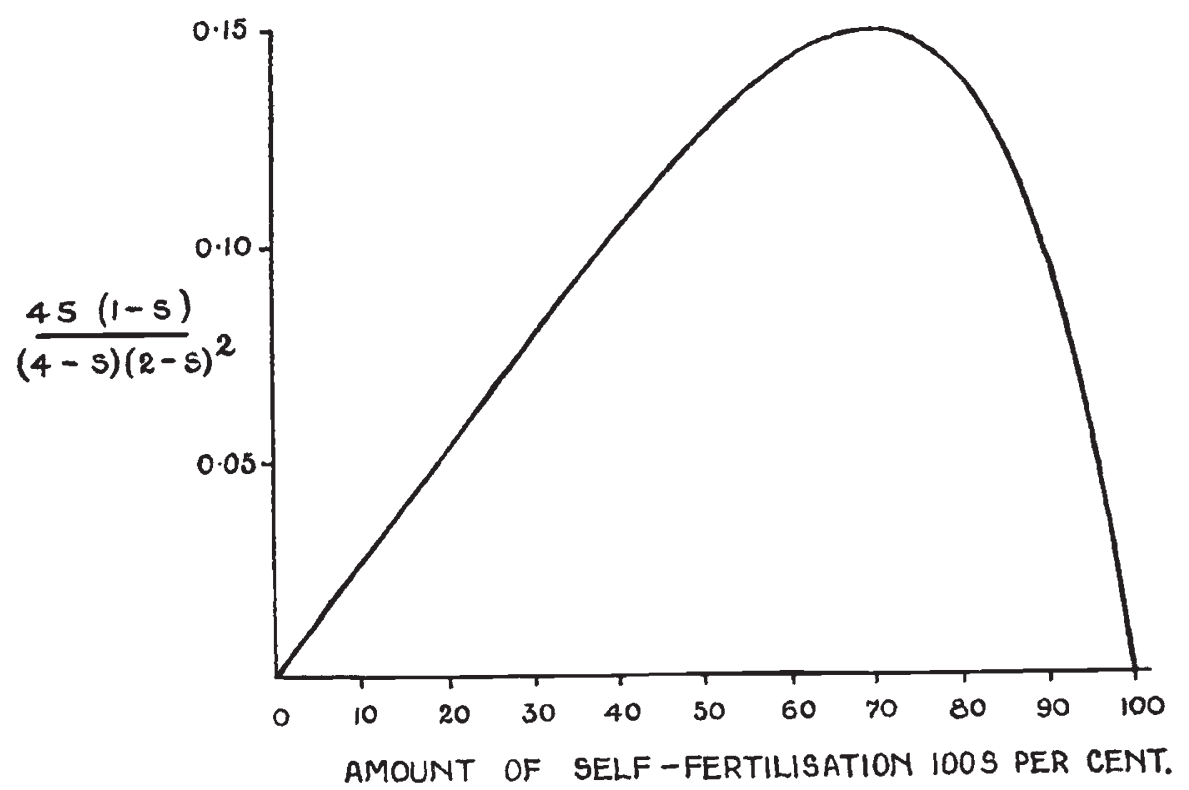

Fig. 1.-Excess of the equilibrium frequency of double homozygotes for two independent loci over the product of the equilibrium frequencies of single homozygotes at these two individual loci expressed as a multiple of the gene frequency product $p(\mathbf{A}) p(a) p(\mathbf{B}) p(b)$ and as a function of the amount of self-fertilisation.

Acknowledgment.-The authors are grateful to Dr H. T. Clifford, University College of Nigeria and formerly of the University of Melbourne, for some initial suggestions and a discussion of relevant botanical aspects of the problem.

\section{REFERENCE}

BenNetT, J. H. 1954. Panmixia with tetrasomic and hexasomic inheritance. Genetics, 39, 150. 